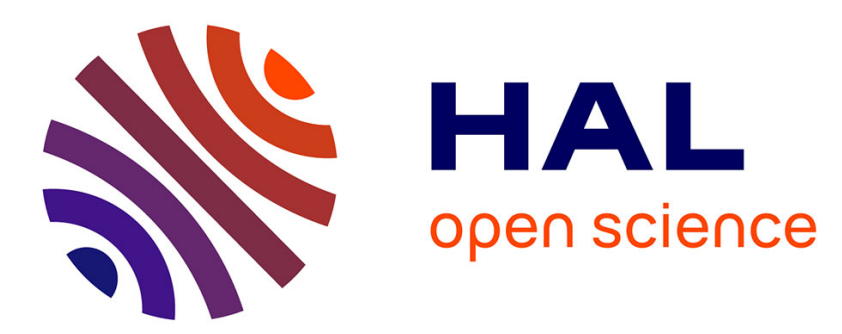

\title{
Mesure de la densité de courant dans une section droite d'un faisceau d'électrons
}

Bernard André

\section{To cite this version:}

Bernard André. Mesure de la densité de courant dans une section droite d'un faisceau d'électrons. Revue de Physique Appliquée, 1966, 1 (2), pp.133-142. 10.1051/rphysap:0196600102013300 . jpa00242698

\section{HAL Id: jpa-00242698 https://hal.science/jpa-00242698}

Submitted on 1 Jan 1966

HAL is a multi-disciplinary open access archive for the deposit and dissemination of scientific research documents, whether they are published or not. The documents may come from teaching and research institutions in France or abroad, or from public or private research centers.
L'archive ouverte pluridisciplinaire HAL, est destinée au dépôt et à la diffusion de documents scientifiques de niveau recherche, publiés ou non, émanant des établissements d'enseignement et de recherche français ou étrangers, des laboratoires publics ou privés. 


\title{
MESURE DE LA DENSITÉ DE COURANT DANS UNE SECTION DROITE D'UN FAISCEAU D'ÉLECTRONS
}

\author{
Par Bernard ANDRÉ, \\ Ingénieur E. N. S. E. E. H. T., Laboratoire de Génie Êlectrique, E. N. S. E. E. H. T., Toulouse.
}

\begin{abstract}
Résumé. - L'auteur propose une méthode électronique rapide générale d'étude expérimentale des caractéristiques de faisceaux d'électrons qui réalise, pour chaque point de la section étudiée, une transposition topologique de la mesure sur un écran oscilloscopique d'observation.

Les principaux résultats d'une étude de la densité électronique et de sa répartition dans un faisceau issu d'un canon triode haute tension expérimental, illustrent une application de cette méthode.
\end{abstract}

Abstract. - A fast electronic method for experimental investigation of the characteristics of electron beams is proposed by the author; this consists of a topological transposition on an oscilloscope screen for observation of the measurement which is to be performed, for every point of the cross-section.

An application of this method is illustrated with the main results of a study of the electron density and of its distribution in a beam from an experimental high voltage triode gun.

Introduction. - La méthode d'investigation que nous avons conçue a été mise en œuvre dans le cadre de recherches sur les sources d'électrons haute tension de moyenne puissance, utilisées en oscillographie cathodique à plusieurs faisceaux [1], [2], [3], [4], [5]. Elle s'est révélée par la suite comme un excellent moven de contrôle dans l'étude des matériaux et composants électroniques soumis à l'action d'un bombardement d'électrons.

Le principe de mesure et d'étude que nous proposons convient lorsqu'il est nécessaire de connaître, instantanément, avec précision, pour tous les points de la section droite analysée, la valeur d'une caractéristique du faisceau. Cette méthode réalise la localisation topologique de la mesure.

L'application de ce principe à l'étude de la densité électronique fait l'objet du présent article [6].

I. Méthodes utilisées pour l'étude de la densité électronique. - La densité électronique $j$ d'un faisceau d'électrons, en un point M d'une surface $(S)$, sécante du faisceau, est égale à la limite du rapport $\mathrm{d} I / \mathrm{d} s$ quand $\mathrm{d} s$ tend vers zéro; $\mathrm{d} I$ est le flux d'électrons qui traverse l'élément de surface $\mathrm{d} s$ de $(S)$ entourant le point $M$.

$$
j=\operatorname{Lim}_{\mathrm{d} s \rightarrow 0} \cdot \mathrm{d} I / \mathrm{d} s .
$$

La mesure de $j$ revient donc à définir un élément de surface $\mathrm{d} s$ aussi petit que possible, et à capter les électrons qui traversent $\mathrm{d} s$. Traditionnellement, un diaphragme d'ouverture connue matérialise $\mathrm{d} s$ tandis qu'une cage de Faraday recueille, au-delà du diaphragme, les électrons le franchissant. En pratique, pour la mesure, l'élément de surface $\mathrm{d} s$ conserve donc, obligatoirement, une dimension finie, d'aire non nulle, de valeur la plus faible possible compatible avec une bonne sensibilité.

A partir de ce principe de mesure, deux types de techniques ont été utilisés pour étudier la répartition de la densité dans une section droite de faisceau :

Déplacement mécanique du diaphragme.

M. Arnaud [7], B. J. Climer [8], par exemple, ont utilisé ce procédé.

Balayage électromagnétique ou électrostatique $d u$ faisceau. - Celui-ci est effectué au droit de la cage de Faraday. La commande peut être manuelle ou automatique. L. Jacob [9], H. Moss [10], M. E. Haine et P. A. Einstein [11], T. Komoda [12] ont mis en œuvre cette technique.

Les divers auteurs que nous venons de citer ont réalisé des dispositifs de mesure qui répondaient aux buts qu'ils poursuivaient. Néanmoins, en général, ces dispositifs ne permettent de réaliser qu'une étude partielle suivant un ou deux diamètres ou bien ils sont longs à mettre en œuvre. Dans tous les cas où les phénomènes observés évoluent en fonction du temps (dérives thermiques ou temporelles) ou lorsqu'il est nécessaire de contrôler périodiquement les caractéristiques du faisceau, la longue durée de la mesure peut rendre impossible l'étude projetée.

Ces deux raisons essentielles nous ont amenés à concevoir, réaliser et mettre au point un ensemble électronique complet original qui, en des temps très brefs, mesure la densité électronique et analyse sa 
répartition suivant l'ensemble d'une section droite d'un faisceau de structure quelconque.

II. Analyseur électronique rapide. Principe. II.1. Principe général (fig. 1). - Cette méthode, entièrement électronique, se rattache au deuxième type : le faisceau est balayé au droit du capteur.

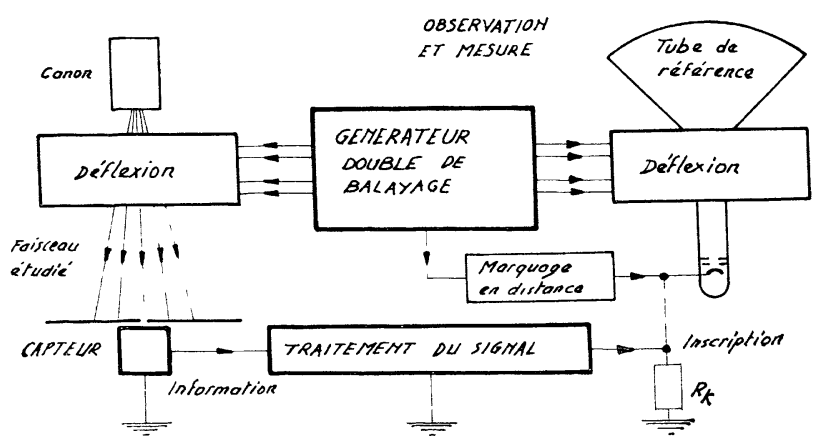

FIg. 1. - Principe de mesure et d'analyse.

A chaque point de la section étudiée, on fait correspondre un " point image ", trace d'un spot sur l'écran d'un tube oscilloscopique de référence. Cette transposition topographique, effectuée en maintenant constant, pour tous les points de la section, le rapport d'homothétie, est réalisée par un générateur double de balayage.

La luminosité du spot varie en fonction de la grandeur caractéristique étudiée : sur l'écran du tube de référence apparaît alors, la représentation globale de la mesure pour l'ensemble de la section.

Sur cette image même peut être superposé le tracé du lieu des points pour lesquels la grandeur caractéristique présente ou bien dépasse une certaine valeur, ou bien encore est comprise entre deux valeurs.

Ces deux dernières fonctions - représentation globale et localisation - sont réalisées par un ensemble de circuits qui transforment le signal recueilli par le capteur. Dans le cas de la mesure de la densité électronique, nous décrirons au paragraphe III la solution que nous avons retenue.

L'organe essentiel commun à toute mesure réalisée ainsi est le générateur double de balayage.

II.2. Générateur double de balayage [6]. Le principe décrit précédemment exige la réalisation, sur deux faisceaux distincts d'électrons, de deux halayages homothétiques qui satisfassent aux conditions supplémentaires suivantes :

- amplitude de balayage du faisceau étudié réglable ;

- nombre de rayons explorés important (400), afin d'obtenir un tracé fin et précis de la carte de répartition ;

- étude complète rapide d'une répartition : le tracé d'une courbe équidensité est réalisé en $1 / 50^{\mathrm{e}}$ de seconde ;

- déflexion magnétique : l'amplitude du balyage est moins affectée par les variations de la haute tension d'accélération qui, dans des études systématiques, peuvent couvrir un large domaine.

Le dispositif réalisé est entièrement électronique et fournit, pour chacun des deux faisceaux, orthogonalement à l'axe de propagation, un champ magnétique qui, pendant un temps $\tau_{1}, 400$ fois par cinquantième de seconde, croît linéairement en fonction du temps de zéro à une valeur maximale $H_{0}$ et qui prend, à chaque balayage, des orientations successives décalées de grade en grade.

Ce champ est obtenu, avec une bonne approximation, en composant deux champs perpendiculaires $\mathbf{H}_{x}$ et $\mathbf{H}_{y}$ (fig. $2 a$ ), d'orientations fixes $\mathrm{O}_{x}$ et $\mathrm{O}_{y}$, perpendiculaires à l'axe de propagation $\mathrm{O}_{z}$ des électrons et tels que :

$$
\begin{aligned}
& \mathbf{H}\left\{\begin{array}{l}
{\left[\begin{array}{l}
\overline{H_{x}}=H_{0} \cos \Omega t .\left(t-t_{n}\right) \\
\overline{H_{y}}=H_{0} \sin \Omega t .\left(t-t_{n}\right) \\
\text { pour } t_{n}<t<t_{n}+\tau_{1}
\end{array}\right.} \\
{\left[\begin{array}{l}
\overline{H_{x}}=\bar{H}_{y}=0 \\
\text { pour } t_{n}+\tau_{1}<t<t_{n+1}
\end{array}\right.}
\end{array}\right. \\
& \text { Valeurs numériques } \\
& F=2 \pi / \Omega=50 \mathrm{~Hz} \\
& \tau_{1}=25 \times 10^{-6} \text { seconde } \\
& t_{n+1}-t_{n}=\tau=50 \times 10^{-6} \mathrm{~s} .
\end{aligned}
$$

Le champ $\mathbf{H}_{x}$, ou le champ $\mathbf{H}_{y}$, est lui-même la résultante de deux champs opposés produits par le passage des courants

$$
\left[\begin{array}{l}
i_{\left(A_{1}+B_{1}\right) x}=i_{0}(1+\alpha \cos \Omega t) .\left(t-t_{n}\right) \\
i_{\left(A_{2}+B_{2}\right) x}=i_{0}(1-\alpha \cos \Omega t) .\left(t-t_{n}\right) \\
\text { pour } t_{n}<t<t_{n}+\tau_{1}
\end{array}\right.
$$

et

$$
\left[\begin{array}{l}
i_{\left(A_{1}+B_{1}\right) x}=i_{\left(A_{\mathbf{z}}+B_{y}\right) x}=0 \\
\text { pour } t_{n}+\tau_{1}<t<t_{n+1}
\end{array}\right.
$$

dans deux bobines de déflexion identiques, $\left(A_{1}+B_{1}\right)_{x}$ et $\left(A_{2}+B_{2}\right)_{x}$, constituées chacune de deux demibobines identiques $\left(A_{1}\right)$ et $\left(B_{1}\right)$, et $\left(A_{2}\right)$ et $\left(B_{2}\right)$, d'axe $\mathrm{Ox}$ et placées symétriquement par rapport à l'axe $\mathrm{Oz}$ (fig. 2b).

Le champ résultant $\overline{H_{x}}$ a alors pour valeur

$$
\left[\begin{array}{l}
\overline{H_{x}}=2 K i_{0} \alpha \cos \Omega t\left(t-t_{n}\right) \\
\text { pour } t_{n}<t<t_{n}+\tau_{1} \\
\text { et } \overline{H_{x}}=0 \\
\text { pour } t_{n}+\tau_{1}<t<t_{n+1}
\end{array}\right.
$$

ce qui correspond bien à la valeur recherchée (1) avec

$$
H_{0}=2 K i_{0} \alpha .
$$


L'amplitude du balayage est proportionnelle à $H_{0}^{\prime}=\tau_{1} H_{0}=2 K i_{0} \tau_{1} \alpha . K, \tau_{1}, i_{0}$ étant constants, il faut, pour faire varier à volonté l'amplitude de balayage du faisceau étudié, agir sur $\alpha$ donc, sur l'amplitude du signal modulateur $(50 \mathrm{~Hz})$.
La réalisation est conforme au schéma-bloc représenté sur la figure 3 .

Remarque: Un dispositif électronique complémentaire permet le marquage en distance de l'image.

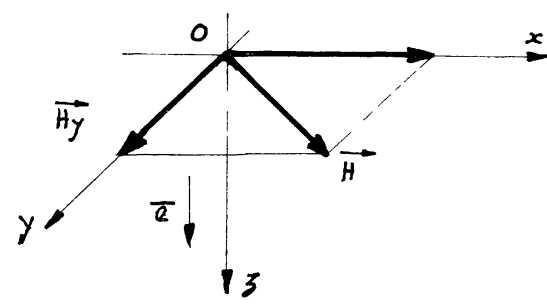

a

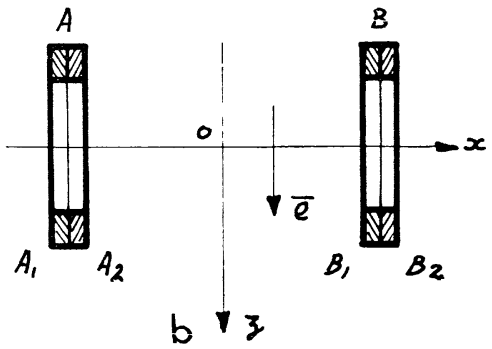

Fig. 2. - a) Production du champ de balayage $\mathbf{H}$ par composition de deux champs perpendiculaires $\mathbf{H}_{x}$ et $\mathbf{H}_{\boldsymbol{v}}$. b) Production du champ $\mathbf{H}_{x}$.

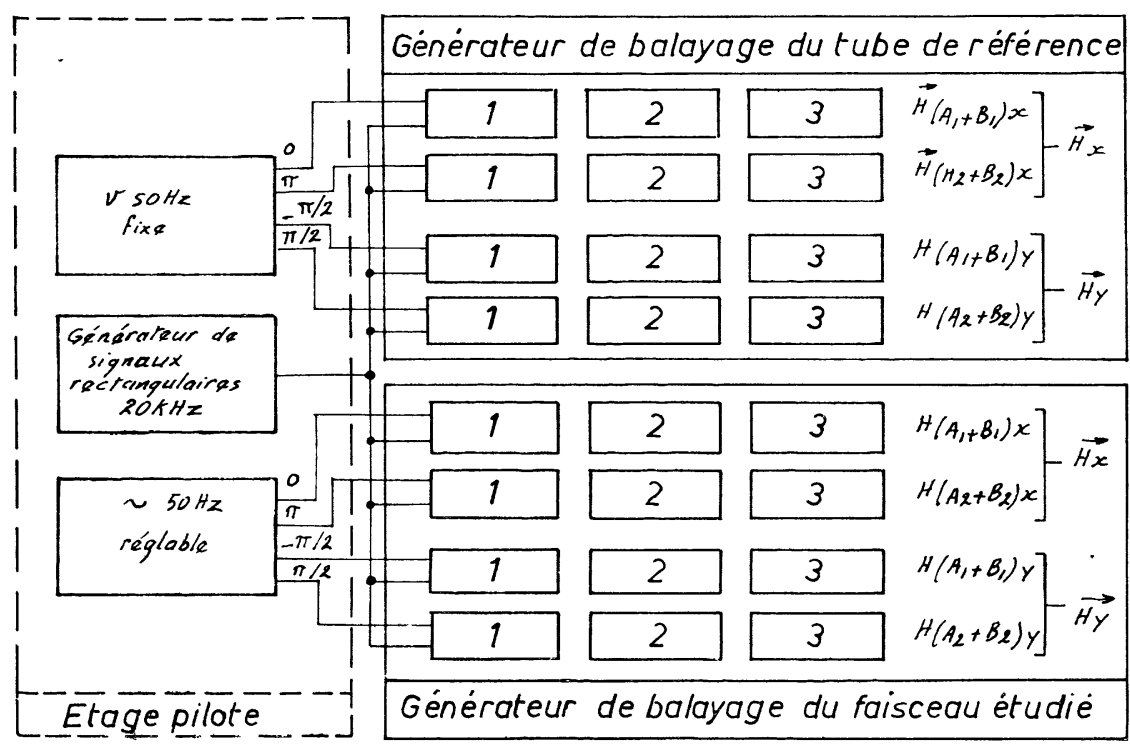

Fig. 3. - Schéma-bloc du générateur double et balayage.

1 : Formation du signal.

2: Amplification.

3 : Bobines de déflexion.

Les indications $0, \pi / 2 \ldots$ donnent les phases des différents signaux $50 \mathrm{~Hz}$ fournis par l'étage pilote.

III. Application de ce principe à la mesure de la densité électronique et à l'étude de sa répartition dans l'ensemble de la section droite d'un faisceau d'électrons. - Dans le cas de la mesure de la densité électronique, le schéma de principe est représenté sur la figure 4 .

Le capteur est une cage de Faraday fixe qui est placée au-delà d'un diaphragme d'ouverture connue $\mathrm{d} s$ et qui fournit un signal $i(t)$ proportionnel à chaque instant à la densité électronique moyenne de la plage du faisceau qui se présente au droit du diaphragme. La valeur ainsi obtenue est d'autant plus proche de la valeur $j$ au centre du diaphragme que $\mathrm{d} s$ est petite $(\S \mathrm{I})$. On peut fixer l'erreur maximale commise en fonction du " profil " de répartition obtenu [6].

Le dispositif permet principalement deux mesures $[13]$ :

Observation qualitative de la répartition globale de la densité électronique dans l'ensemble de la section étudiée. - Elle est réalisée simplement en modulant la luminosité du spot du tube de référence par le signal $i(t)$ convenablement amplifié.

Mesure quantitative en tous les points. - La méthode consiste à réaliser sur l'écran d'observation du tube de référence le tracé du lieu des points d'égale 


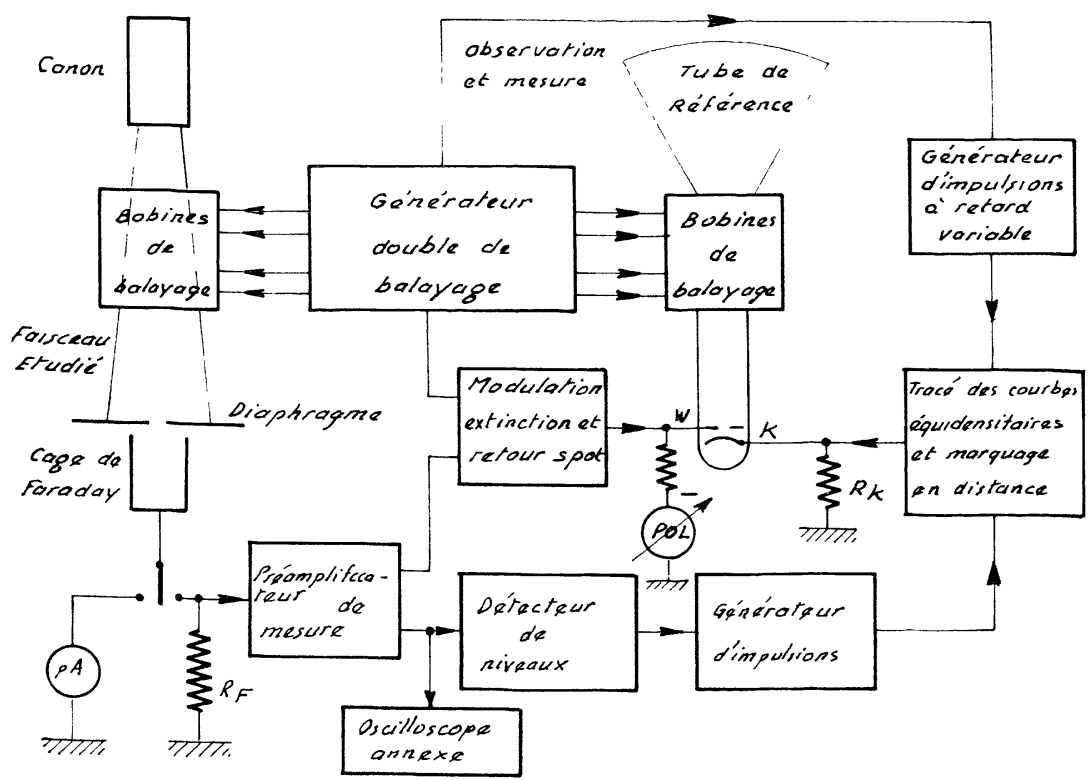

Fig. 4. - Schéma de principe de l'analyseur électronique rapide.

densité électronique sous forme de courbes équidensité se superposant ou non à l'image obtenue précédemment. Le signal $i(t)$ est, après amplification, appliqué à un circuit détecteur de niveaux dont le rôle apparaît sur la figure 5 .

A chaque passage du signal au niveau choisi $(k i)_{0}$ le détecteur déclenche et une impulsion de marquage est fournie par le générateur d'impulsions à

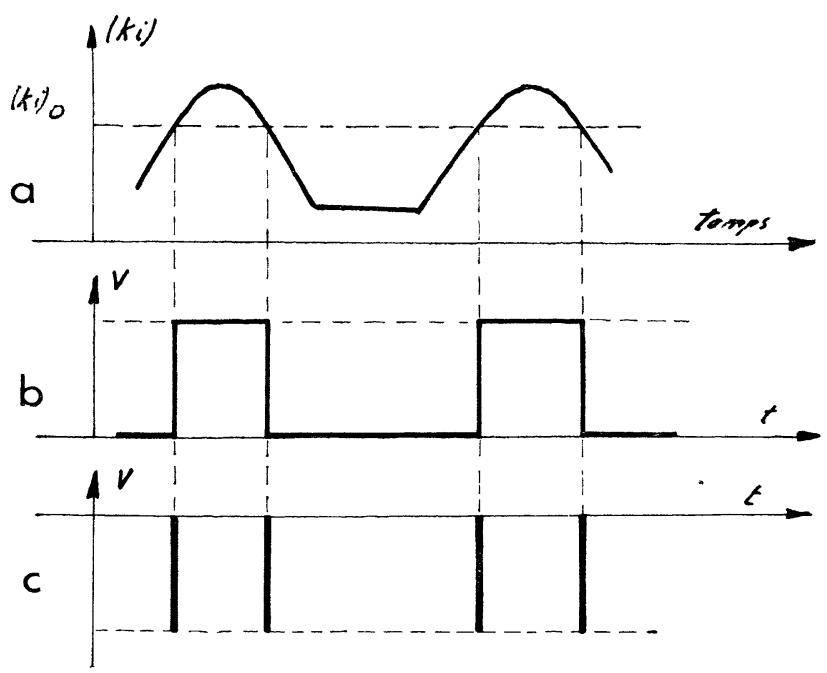

FIG. 5. - Mesure quantitative : principe de fonctionnement du détecteur de niveaux et du générateur d'impulsions.

a) Signal issu de l'amplificateur proportionnel à $j$.

b) Signal fourni par le détecteur de niveaux.

c) Impulsions de marquage fournies par un générateur. la cathode du tube de référence. Il apparaît donc en surbrillance, sur l'écran d'observation, des courbes qui sont l'image du lieu des points de la section pour lesquels la densité a la valeur définie par $i_{0}$.

L'énoncé du principe du dispositif électronique de mesure montre les rôles importants et complémentaires que jouent les circuits d'amplification et de détection de niveaux.

III.1. Dispositif Électronique de mesure. III.1.1. Chaîne d'amplification. - L'expérience nature du capteur, type de balayage - impose un certain nombre de conditions concernant la bande passante, le gain, le rapport signal sur bruit. Elle nécessite, en outre, que les liaisons entre les étages amplificateurs successifs soient directes.

En nous plaçant dans le cas le plus défavorable, nous pouvons évaluer une limite supérieure de la bande passante nécessaire. Elle dépend, d'une part, de la vitesse de balyage ou plus précisément du temps $\tau_{1}(1)$, et d'autre part, du rapport : diamètre de l'ouverture de la cage de Faraday $d_{\mathrm{F}}$ sur le rayon de la section $R_{\mathrm{s}}$. Elle s'exprime par la formule approchée $R_{\mathrm{S}} / 4 d_{\mathrm{F}} \tau_{1}=\left(R_{\mathrm{S}} / d_{\mathrm{F}}\right) 10^{4} \mathrm{~Hz}$. Par exemple pour $d_{\mathrm{S}} / R_{\mathrm{F}}=10^{-2}$ la bande passante nécessaire sera de 0 à $1 \mathrm{MHz}$.

La formulation d'une limite acceptable au rapport signal sur bruit est plus délicate. Elle dépend de la densité minimale qu'on souhaite pouvoir analyser et de l'ouverture du diaphragme. De toute évidence, il faut s'attacher à diminuer le plus possible le bruit de fond de l'amplificateur compte tenu du gain et de la bande passante nécessaire [14]. C'est alors plutôt la connaissance du bruit de fond qui fixera, 
en fonction de la densité maximale et de la surface de la section, les conditions de la mesure (ouverture du diaphragme, gains) et les limites de sensibilité [6].

III.1.2. Détecteur à niveau de seuil variable. Réaliser la mesure complète et précise nécessite que ce circuit satisfasse à trois conditions importantes :

- le niveau détecté doit être indépendant de la fréquence du signal : ce circuit est à large bande passante ;

- le niveau détecté doit être variable : cette fonction permet la mesure de la densité en tous les points :

- enfin, il faut que les niveaux du signal $(k i)_{0}$ et $(k i)_{\mathrm{F}}$ à l' " ouverture " et à la "fermeture " du détecteur soient sensiblement identiques, que le signal croisse ou décroisse.

La figure 6 montre de quelles façons la mesure peut être faussée :

- soit par absence de détection au niveau choisi (points $\mathrm{M}_{2}, \mathrm{M}_{3}, \mathrm{M}_{4}$ )

- soit par déclenchement à la fermeture à un niveau $(k i)_{\mathrm{F}}$ différent de $(k i)_{0}$ (points $\mathrm{M}_{5}$ ). Il importe donc de minimiser l'écart $(k i)_{0}-(k i)_{\mathrm{F}}$ ou miєux encore, le rapport $(k i)_{0}-(k i)_{\mathrm{F}} J(k i)_{\max },(k i)_{\max }$ étant l'amplitude maximale du signal que peut analyser le détecteur.

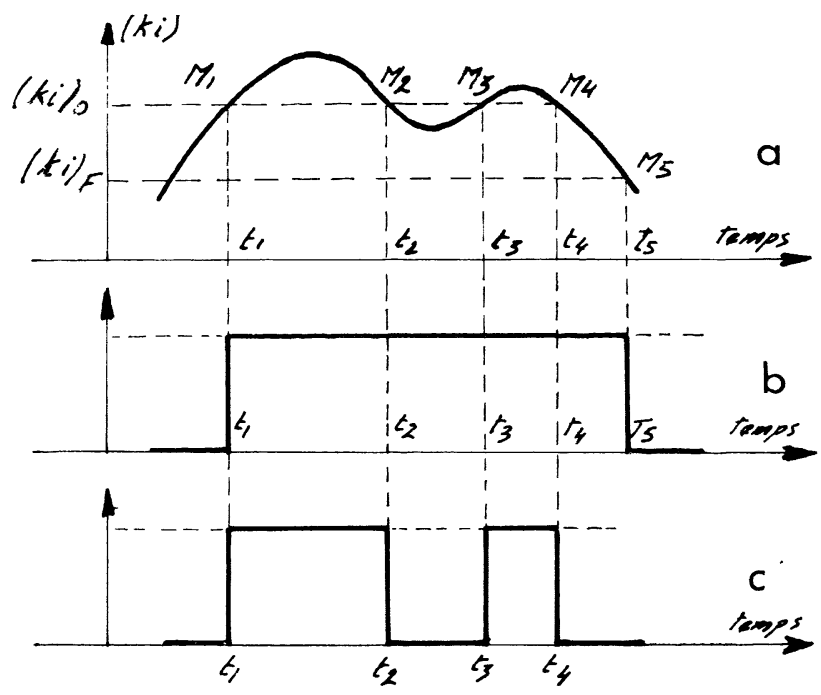

FIG. 6. - Détecteur de niveaux.

a) Signal d'entrée, niveau d'ouverture $(k i)_{0}$, niveau de fermeture $(k i)_{\mathrm{F}}$ - $-b$ ) Signal de sortie, détecteur classique. - c) Signal de sortie, détecteur idéal.

III.1.3. Le dispositif de mesure. - Sur la figure 7 apparaît bien le rôle joué par chacun des circuits

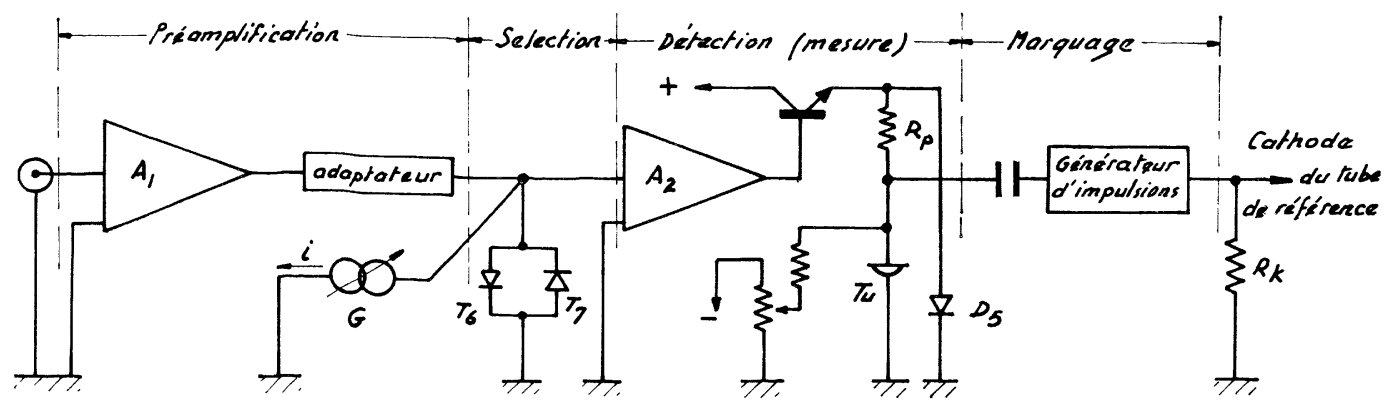

FIG. 7.

qui constituent l'ensemble du dispositif de mesure :

- préamplification réalisée par le circuit $A_{1}$ qui est suivi d'un circuit adaptateur 6 gammes de mesure, gain variant de $10^{6}$ à $1 / 310^{8} \mathrm{ohm}$ pour des bandes passantes allant de 2400 à $300 \mathrm{kHz}$, amplitude maximale de sortie 4 volts);

- sélection, à l'aide des diodes $\mathrm{T}_{6}$ et $\mathrm{T}_{7}$ et du générateur étalonné de courant continu réglable $\mathrm{G}$, d'une partie du signal dont la valeur moyenne correspond au niveau détecté ;

- détection (mesure) par la diode tunnel $\mathrm{T}_{u}$, précédée de l'amplificateur $\mathrm{A}_{2}$ (diminution de $(k i)_{0}-(k i)_{\mathbf{F}}$ mesurée à l'entrée) et protégée par le circuit résistance $R_{p}$ et diode $\mathrm{D}_{5}$ (augmentation de $\left.(k i)_{\max }\right)$;

- tracé des courbes équidensité sur l'écran d'observation grâce à des impulsions fournies par un générateur synchronisé sur les fronts de commutation de la diode tunnel de détection et transmises à la cathode du tube de référence.

III.2. Performances. - Un certain nombre de causes d'erreur peuvent intervenir dans l'application de la méthode de mesure décrite précédemment [6].

Certaines sont dues au collecteur d'électrons ou au faisceau lui-même :

- dimension finie de l'ouverture de la cage de Faraday ;

- non monocinétisme des électrons.

D'autres proviennent du balayage :

- non uniformité du champ ;

- non linéarité des courants qui parcourent les bobines de déflexion.

D'autres encore, peuvent affecter directement la mesure : 
- bandes passantes des divers circuits insuffisantes ;

- écart entre les niveaux détectés $(k i)_{0}$ et $(k i)_{\mathrm{F}}$;

- retard entre l'instant de passage du point de la section pour lequel la densité à la valeur correspondant au niveau de détection choisi et l'instant d'application au wehnelt du tube de référence de l'impulsion de marquage correspondante.

Chaque élément de montage a été étudié de façon à en minimiser l'influence. Les divers circuits ont été étalonnés séparément. Enfin, des mesures ont été réalisées sur différentes structures de faisceaux et comparées aux résultats obtenus au moyen d'une méthode classique. Cette dernière précise, mais très longue à mettre en œuvre, consiste à déplacer, dans la section étudiée, manuellement et mécaniquement le diaphragme d'ouverture de la cage de Faraday et à mesurer, point par point, le courant recueilli. Les résultats obtenus au moyen de ces deux méthodes ne diffèrent, au plus, que de $10 \%$ [6].

En résumé, la réalisation actuelle permet de déceler des courants issus de la cage de Faraday de $5 \mathrm{nA}$ et d'analyser des courants allant de $40 \mathrm{nA}$ à $4 \mathrm{~mA}$.

III.3. Circuits complémentaires. Disposition D'ensemble. - L'ensemble de l'analyseur comprend, en outre, les circuits suivants :

— sources d'alimentation ;

- circuit d'amplification du signal pour la modulation du tube de référence ;

- circuit de blocage du wehnelt pendant le retour à l'origine du spot du tube de référence ;

- circuit de marquage en distance de l'image ;

- circuits destinés au contrôle et au réglage du zéro du préamplificateur et du détecteur de niveaux;

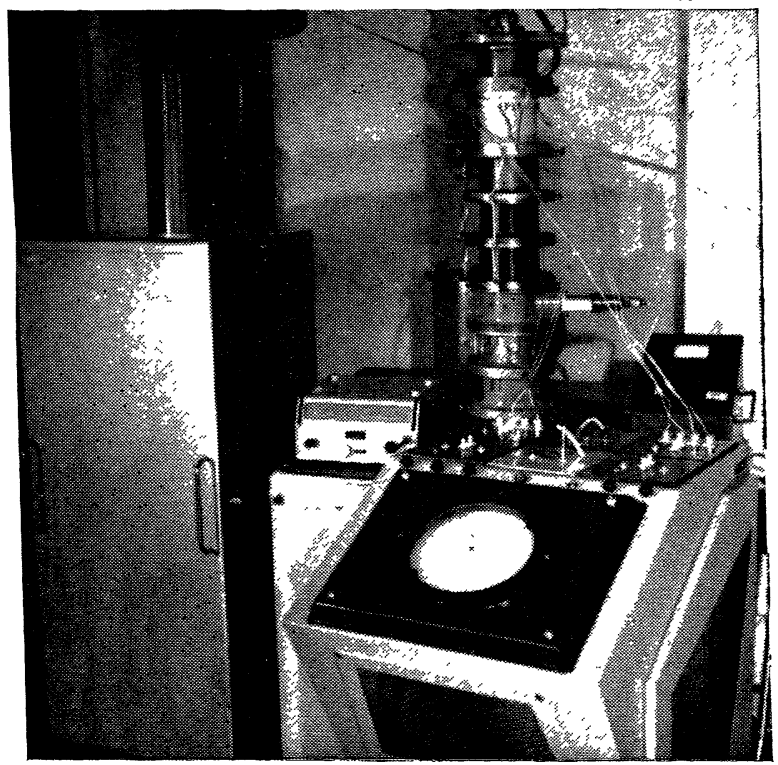

FIG. 8. - Ensemble expérimental.
- circuit permettant le tracé, sur un oscilloscope annexe, de la courbe représentant les variations de $j$ le long d'un rayon quelconque de la section étudiée.

Tous ces circuits, ainsi que ceux décrits précédemment, ont été câblés sur des châssis à glissières et prennent place dans un ensemble qui reçoit aussi le tube de référence (tube récepteur de télévision). La photo de la figure 8 représente l'ensemble du dispositif expérimental : l'analyseur de faisceau est en premier plan.

IV. Étude de la densité électronique et de sa répartition dans une section droite de faisceaux larges d'électrons issus de canons à électrons à haute tension [6]. - IV.1. GRANdeurs CaractéRISTIQUES ET PARAMÈTRES DES FAISCEAUX ET SOURCES D'ÉLECTRONS. - Le fonctionnement d'une source d'électrons est caractérisé par des grandeurs qui s'attachent à la source ou au faisceau produit. Ces grandeurs dépendent des paramètres qui définissent les conditions de mise en œuvre du canon.

IV.1.1. Grandeurs caractéristiques. - Brièvement mentionnées ici, ce sont :

- l'énergie des électrons, qui dépend de leur vitesse de propagation ou de la tension d'accélération ;

- l'angle d'ouverture du faisceau ;

- l'intensité, la brillance, la densité électronique $j$;

- le rendement énergétique du canon $\eta$;

- le rendement $\eta_{j}=j / j_{\text {th }}$ ( $j_{\text {th }}$ est la valeur maximale théorique de la densité [15]) ;

- la pervéance ;

- la position et la dimension du crossover.

Certaines de ces grandeurs sont accessibles directement par le calcul ou par de simples mesures. Pour accéder aux autres, il est nécessaire de connaître la valeur de la densité électronique. D'autre part, dans les deux perspectives d'applications oscillographie cathodique haute tension à plusieurs faisceaux et étude des matériaux et composants électroniques soumis à des bombardements d'électrons - la connaissance concrète de la densité électronique et de ses variations dans une section droite est indispensable.

IV.1.2. Paramètres. - Cette étude porte sur les canons à électrons de type triode à cathode à chauffage direct.

Leur fonctionnement dépend de trois types de paramètres :

- électriques :

tension d'accélération $\overline{V_{\mathrm{a}}}$ (ddp entre l'anode et la cathode),

tension de commande $\overline{V_{\mathrm{G}}}$ (ddp entre le wehnelt et la cathode) ;

- géométriques: toutes les grandeurs qui permettent de caractériser la forme et les dimensions des électrodes et leur disposition les unes par rapport aux autres. On peut ainsi définir : 
$D$ : diamètre de l'ouverture de l'anode,

$d$ : diamètre de l'ouverture du wehnelt,

$h$ : distance cathode-wehnelt,

$H$ : distance cathode-anode.

- physico-chimiques : ils caractérisent la cathode et son émission spécifique (nature de la cathode, température...).

IV.1.3. Canon à électrons expérimental [6]. - Pour contrôler et mesurer les différents paramètres, nous avons réalisé un canon expérimental adapté à une installation à vide entretenu. Pour sa conception, nous nous sommes imposé les conditions suivantes:

- tension d'accélération maximale $50 \mathrm{kV}$;

- réglage sous vide et sous tension des distances $h$ et $H$;

- possibilité de centrer, sous vide et sous tension, la cathode par rapport au wehnelt et l'anode par rapport à l'ensemble cathode-wehnelt ; contrôler ce centrage ;

- possibilité de mesurer la température de la cathode ;

- possibilité de mesurer les distances $h$ et $H$;

- possibilité de refroidir le support de cathode ;

- changement aisé de la cathode, du wehnelt et de l'anode ;

- possibilité d'adjoindre des électrodes supplémentaires $( \pm 5 \mathrm{kV}$ par rapport à la cathode).

Le principe de réalisation est schématisé sur la figure 9 .

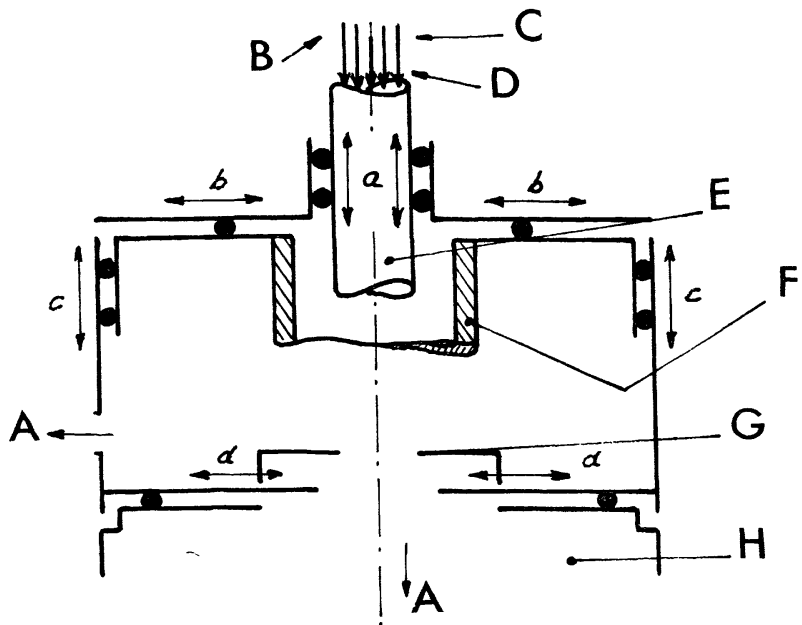

Fig. 9. - Principe de réalisation du canon à électrons expérimental. A. Pompage. $-B$. Refroidissement du support de cathode. $-C$. Chauffage du filament. $D$. Polarisation du wehnelt. - E. Support de cathode. - F. Support du Wehuelt. - G. support d'anode. - $H$. Enceinte à vide entretenu.

Les photographies des figures 10 et 11 donnent un aspect des pièces essentielles.

IV.2. Principaux résultats. - Les recherches expérimentales sur les canons à électrons ont été conduites, à l'aide de l'analyseur de faisceau et du

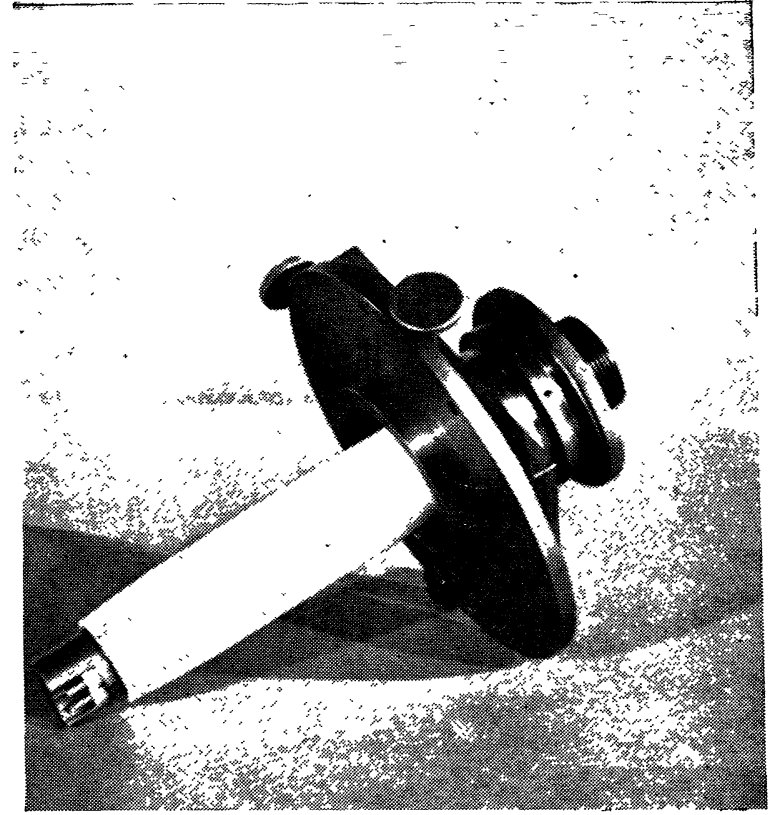

Fig. 10. - Réglage et centrage de la cathode par rapport au wehnelt. Isolement haute tension de l'ensemble cathode-wehnelt.

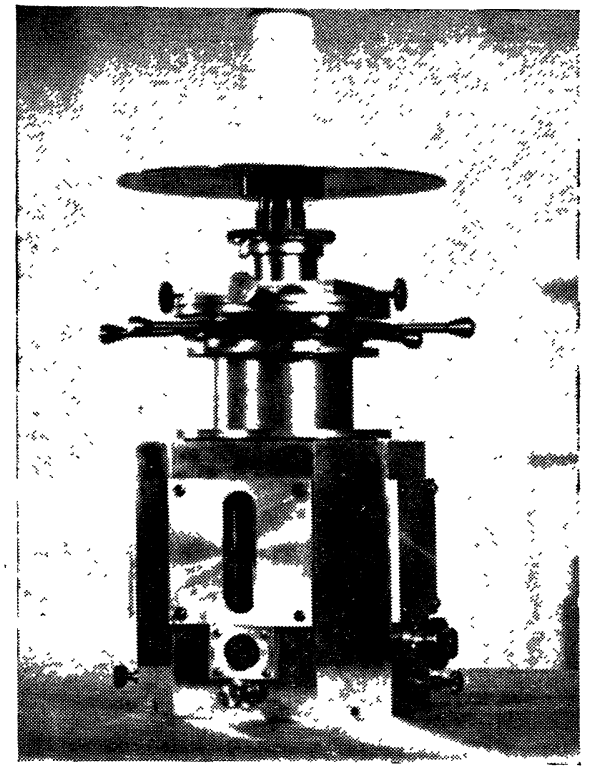

FIg. 11. - Pièce support de l'anode et de l'ensemble cathode-wehnelt : fenêtres de visées, commandes.

canon expérimental, en vue de déterminer les sensibilités de la densité électronique et de sa répartition à la variation des différents paramètres du canon. Nous nous sommes particulièrement attachés à définir les conditions de formation de faisceaux 
larges à maximum annulaire de répartition ou à répartition uniforme.

IV.2.1. Influence de la tension de swehnelt. - Nous avons retrouvé dans cette étude des résultats obtenus par différents auteurs [11], [12], [16] : tous les autres paramètres étant maintenus constants, lorsque $\left|V_{\mathrm{G}}\right|$ diminue, on observe, en général, dans l'évolution du profil de répartition le long d'un diamètre, les différentes étapes suivantes ( $f$ g. 12a, b, $c, d)$. :

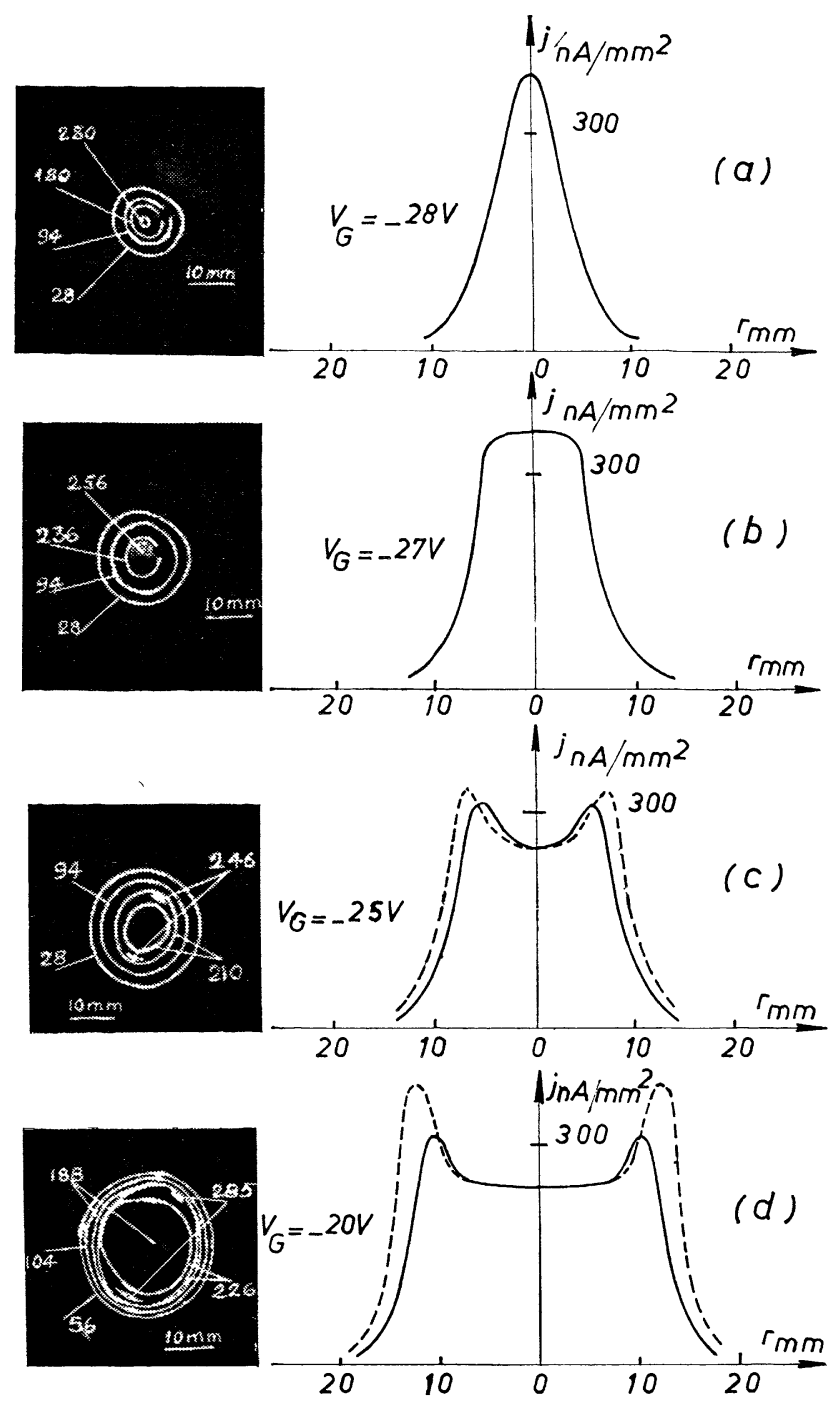

FrG. 12. - Influence de la tension de polarisation $V_{\mathrm{G}}$ sur la répartition de la densité électronique $j$ en fonction de la distance au centre $r$.

a) apparition, pour $V_{\mathrm{G}}=V_{\mathrm{GC}}$ (tension de cut-off), d'un faisceau étroit présentant une répartition classique à un maximum de densité au centre; au fur et à mesure que $\left|V_{\mathrm{G}}\right|$ diminue, la section du faisceau augmente, le maximum augmente; b) pour une seconde valeur critique de $V_{\mathrm{G}}, V_{\mathrm{GM}}$, le maximum au centre atteint la plus forte valeur (fig. 12a);

c) si $\left|V_{\mathrm{G}}\right|$ diminue encore la section du faisceau augmente toujours et le profil de répartition s'écarte de plus en plus de la représentation formulée par D. B. Langmuir ; le maximum au centre n'augmente plus ou diminue : on tend vers une répartition uniforme dans la région centrale ;

d) une troisième valeur critique $V_{\mathrm{GU}}$ est déterminée quand la zone centrale à répartition quasiuniforme a atteint la surface maximale et qu'il n'y a pas encore de maximum annulaire périphérique (fig. 12b);

e) enfin, quand $\left|V_{\mathrm{G}}\right|$ diminue encore, la densité de la zone centrale diminue, tandis que celle d'une zone périphérique annulaire augmente sensiblement : on tend vers la formation d'un faisceau creux (fig. $12 c$ et $12 d$ ).

Ces différentes phases sont d'autant plus nettes que la distance cathode-wehnelt $h$ est petite ou que l'ouverture du wehnelt $d$ est grande par rapport à la surface de la cathode. Elles se déroulent sensiblement dans les mêmes conditions avec différents types de wehnelt et avec des cathodes de métal et de forme différents (fils ou rubans de tungstène, tantale, molybdène).

Néanmoins, les deux dernières phases ne se produisent pas lorsque l'ouverture du wehnelt est plus petite que la surface de cathode.

Une explication qualitative de ces phénomènes peut être déduite à partir des travaux de différents auteurs [17], [11], [12], [16]. Elle s'appuie sur le fait que la surface émissive augmente lorsque $\left|V_{\mathrm{G}}\right|$ diminue et qu'il y a une distorsion du champ au niveau de la cathode [6].

Les phénomènes que nous venons de décrire nous ont servi de critère dans l'étude de l'influence des variations des autres paramètres. Les principaux résultats rendent compte de l'étude des variations de la valeur singulière $V_{\mathrm{GM}}$. En effet, d'une part, $V_{\text {GM }}$ peut être mesurée sans ambiguité (observation d'un maximum), d'autre part, $V_{\mathrm{GM}}$ correspond à un fonctionnement intéressant de la source (densité maximale au centre) et enfin, $V_{\mathrm{GM}}$ permet d'accéder aux autres valeurs singulières.

IV.2.2. Influence de la tension d'accélération $V_{\mathrm{a} .}$ Tous les autres paramètres étant maintenus constants, lorsque la tension $V_{\mathrm{a}}$ varie, les différentes phases décrites dans le paragraphe précédent se produisent : à une diminution de $\left|V_{\mathrm{G}}\right|$ correspond une augmentation de $V_{\text {a }}$.

Pour caractériser l'influence réciproque de $V_{\mathrm{G}}$ et de $V_{\mathrm{a}}$ sur la répartition de la densité d'un canon à électrons de structure donnée, nous pouvons tracer une courbe qui, dans un plan $\left(V_{\mathrm{a}}, V_{\mathrm{G}}\right)$, fait correspondre à chaque tension $V_{\mathrm{a}}$ la valeur $V_{\mathrm{GM}}$ - ou la valeur $V_{\mathrm{GU}}$ - correspondante (fig. 13).

Le plan représentatif est alors divisé en deux ou 


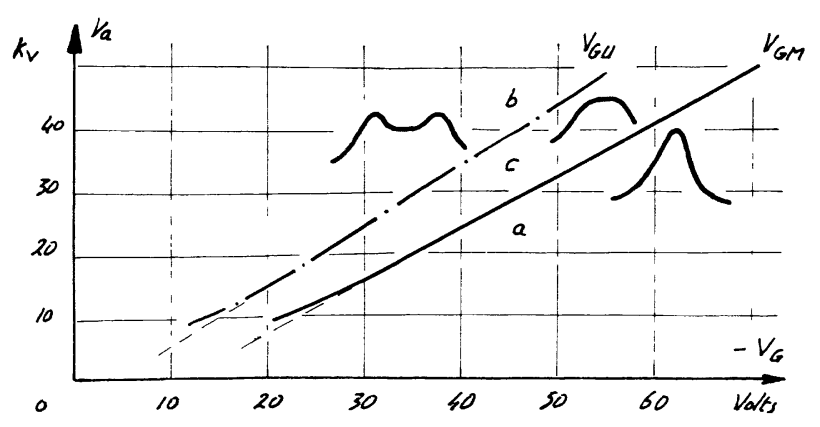

FIG. 13. - Représentation de la répartition en fonction de $V_{\mathrm{G}}$ et de $V_{\mathrm{a}} . \mathrm{T}_{\mathrm{K}}=2700^{\circ} \mathrm{K}, d=4 \mathrm{~mm}, D=6 \mathrm{~mm}$, $h=3 \mathrm{~mm}, H=40 \mathrm{~mm}$. Filament de tungstène et wehnelt hémisphérique.

trois régions caractéristiques de la répartition de densité :

— zone a : répartition à allure gaussienne ;

— zone $\mathrm{b}$ : répartition à maximum périphérique ;

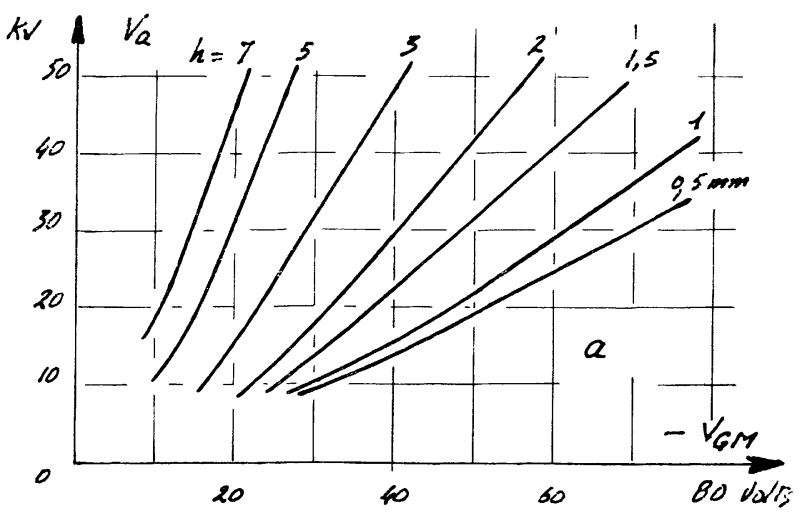

- zone $\mathrm{c}$ : zone de transition à répartition quasiuniforme.

Dans les différentes études que nous avons réalisées, les points représentatifs $\left(V_{\mathrm{a}}, V_{\mathrm{GM}}\right)$ ou $\left(V_{\mathrm{a}}, V_{\mathrm{GU}}\right)$ sont alignés avec une bonne approximation pour $V_{\mathrm{a}}>15$ à $25 \mathrm{kV}$. Pour des tensions d'accélération inférieures, la courbe représentative s'écarte de plus en plus de la droite. Pour de faibles tensions $V_{\mathrm{a}}$ il peut ne pas exister de valeurs de $V_{\mathrm{G}}$ qui fassent apparaître la répartition à maximum périphérique.

La pente de la droite et, en général, l'allure des courbes représentatives $V_{\mathrm{a}}\left(V_{\mathrm{GM}}\right)$ ou $V_{\mathrm{a}}\left(V_{\mathrm{GU}}\right)$ sont fonction des autres paramètres.

IV.2.3. Influence de la distance cathode-svehnelt $h$. - Les résultats obtenus pour différentes valeurs des autres paramètres présentent une bonne similitude. Le faisceau de courbes de la figure $14 a$ montre, sur un exemple, l'influence de $h$ sur la répartition de la densité.

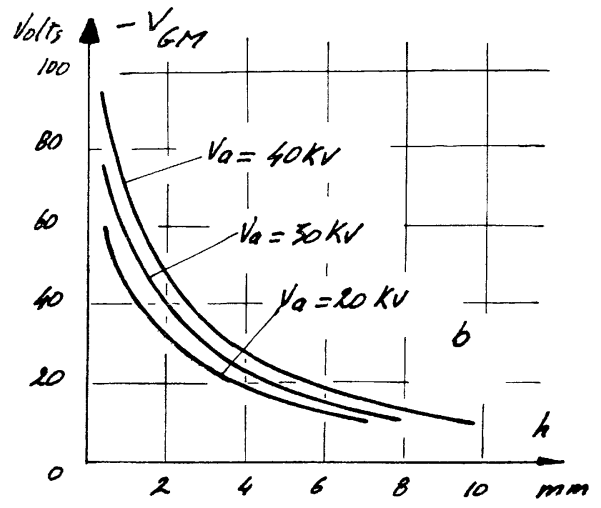

FIG. 14. - a) Influence de la distance cathode-wehnelt $h$ sur la répartition de la densité.

b) Variations de $V_{\mathrm{GM}}$ en fonction de $h .\left(T_{\mathrm{K}}=2150^{\circ} \mathrm{K}, d=3 \mathrm{~mm}, D=6 \mathrm{~mm}, H=40 \mathrm{~mm}\right.$.)

A tension d'accélération constante, les variations de $V_{\mathrm{GM}}$ en fonction de $h$ ont une allure hyperbolique (fig. 14b).

IV.2.4. Influence de la température de la cathode. Si les variations de la température $T_{K}$ ont beaucoup d'influence sur le courant total du faisceau $I_{\mathrm{F}}$ et sur la densité maximale, elles ont relativement peu d'influence sur la répartition de densité. Elle est sensible, néanmoins, lorsque l'écart thermique est important. Cette influence est alors caractérisée par une translation des courbes $V_{\mathrm{a}}\left(V_{\mathrm{GM}}\right)$ parallèlement à l'axe des $V_{\mathrm{GM}}$ (fig. 15).

Conclusion. - Les quelques résultats principaux, mais partiels, que nous présentons ici ne se prêtent pas à une formulation globale rigoureuse non seulement parce que les paramètres qui interviennent sont nombreux mais parce que certains, comme par exemple la forme des électrodes, n'offrent aucune possibilité de mise en équation générale.
Néanmoins, les lois qualitatives que les exemples choisis mettent en évidence demeurent générales et, le plus souvent, sont suffisantes pour guider la mise au point des sources de faisceaux larges d'électrons.

L'analyseur rapide de faisceaux que nous venons d'étudier brièvement rend possible, dans sa réalisation actuelle, l'étude complète de la répartition de la densité électronique dans une section quelconque de bon nombre de faisceaux larges d'électrons, d'intensité totale minimale $10 \mu \mathrm{A}$, de tension d'accélération maximale $100 \mathrm{kV}$ et dont la surface d'étude peut varier de 0,5 à $20 \mathrm{~cm}^{2}$ environ.

Il peut assurer un contrôle continu ce qui permet d'observer des phénomènes de dérives thermique (dilatation des supports d'électrodes) ou temporelle (vieillissement de cathodes...).

Il s'adapte également à l'étude de sources impulsionnelles d'électrons sous réserve que le canon fonctionne pendant au moins $25 \mu$ s et que les impulsions de déblocage soient synchronisées avec le balayage (fréquence $20,10,5 \ldots, \mathrm{kHz}$ pour 400 , 


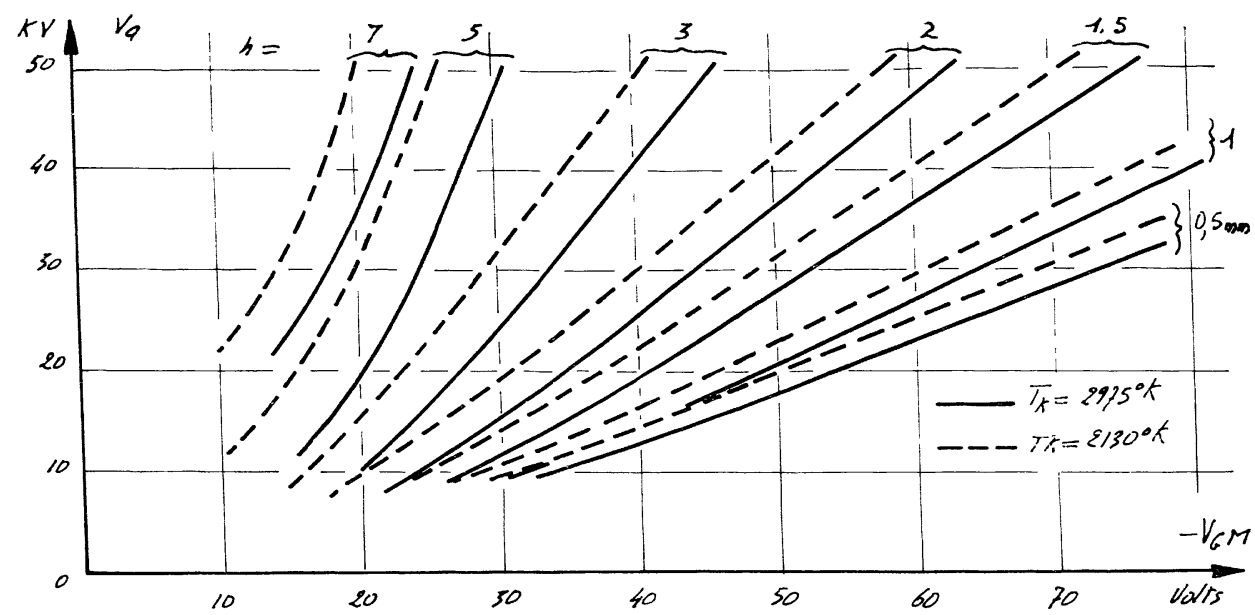

FIG. 15. - Influence de la température de la cathode $T_{\mathrm{K}}$ sur la répartition de la densité électronique.

$200,100, \ldots$ rayons explorés ou bien fréquences plus basses avec des retards de $50,100, \ldots$ microsecondes introduits au déclenchement du canon à chaque cycle complet de balayage).

Les performances de l'analyseur peuvent être améliorées ou la méthode de mesure adaptée à des problèmes particuliers soit, en modifiant la nature du capteur ou en augmentant sa sensibilité (multiplicateur d'électrons), soit, en changeant la fréquence de balayage, le nombre de rayons explorés ou le type même de balayage.
Dans le cadre le plus général de recherches en fiabilité (physique des défaillances) cette méthode est particulièrement bien adaptée à la mesure précise et continue de la densité électronique et de sa répartition dans la section utile de faisceaux d'électrons utilisés dans les études de la dégradation de matériaux diélectriques et de composants semiconducteurs soumis à irradiation.

Manuscrit reçu le 28 avril 1966.

\section{BIBLIOGRAPHIE}

[1] OLLE (J.), Étude et réalisation d'un oscillographe à quatre faisceaux et à haute tension. Thèse Docteur-Ingénieur, Toulouse, 20 février 1954.

[2] Lagasse (J.), Fert (Ch.) et Clot (J.), Perfectionnements apportés aux oscillographes cathodiques à plusieurs faisceaux. Brevet CNRS no 1162163, 8 avril 1958 et complément no 71037 , 23 mars 1959.

[3] Lagasse (J.), Fert (Ch.) et Clot (J.), Une conception nouvelle d'oscillographe cathodique à voies multiples. C. R. Acad. Sc., Paris, 1957, 244, 2148.

[4] Queau (A.), Étude et réalisation d'un tube oscillographique monocanon permettant l'enregistrement simultané de plusieurs phénomènes. Thèse Doctorat $3^{\text {ème }}$ cycle, spécialité Électronique, Toulouse, 31 octobre 1961.

[5] Clot (J.), Lagasse (J.) et Queau (A.), Sur la réalisation d'un tube oscilloscopique monocanon permettant l'observation simultanée de deux phénomènes. C. R. Acad. Sc., Paris, 1961, 253, 2893-2895.

[6] André (B.), Thèse Doctorat ès Sciences, à paraître.

[7] Arnaud (M.), Étude et mise au point d'une machine à essayer les canons avec enregistrement automatique de la densité de courant. Revue technique C.F.T.H., Paris, $\mathrm{n}^{\circ}$ 32, février 1960.

[8] Crimer (B. J.), Electron Current Densities and Transverse Velocities in Pierce Emission Systems. J. Electron Control GB, 1962, 13, $\mathrm{n}^{\circ} 5$, 385-400.
[9] $\mathrm{J}_{\mathrm{AcoB}}$ (L.), Electron distribution in Electron optically focused Electron Beams. Phil. Mag., 1939, 28, no 186,80.

[10] Moss (H.), Measurements of the Limiting Image Current Density produced by Electron gun of rotational Symmetry. J. Electronics, 1961, 10, 341.

[11] Haine (M. E.) et Einstein (P. A.), Characteristics of the hot Cathode Electron Microscope Gun. British J. Appl. Physics, 1952 , 3, 40-46.

[12] Komoda (T.), Performances of the Gun with a Re-entrant Shaped Wehnelt Cylinder. J. Electron Microscopy, 8, 8-12.

[13] André (B.), Sur un dispositif électronique de détermination de la répartition de la densité de charges dans la section droite d'un faisceau électronique. C. R. Acad. Sc., Paris, 1962, 254, 3505.

[14] Giralt (G.), Martin (J. C.) et Chauvet (F.), Étude d'un amplificateur continu et à liaisons directes à bande passante élevée. Rev. Gén. électronique, février 1965, no 219.

[15] Langmuir (D. B.), Theoretical limitation of cathode ray tubes. Proced I. R. E., 1937, 25, 977-999.

[16] Sugata (E.) et Hamada (H.), Studies of Electron gun for electron microscope by resistance network analog. J. Electron microscopy, 1960, 8, 4-7.

[17] Moss (H.), Electron gun of the cathode ray tube. $J$. Bristh instit. radio engineers, 1945, part. 1, vol. $5, \mathrm{n}^{0}$ 1, 10-22; part. 2, juin 1946. 